\title{
Modification Method of Tooth Profile of Locomotive Traction Gear Based on Rodent Arm Variation
}

\author{
Lei Lei', Haichun Ding ${ }^{1 *}$, Liang Xuan², Meichao Qin¹, Siyuan Tian¹, Yunan Sui \\ 1 Department of Mechanical Drive, School of Mechanical Engineering, Dalian Jiao Tong University, \\ 116000 Dalian, P. O. B. 289\#, China \\ 2 Department of Mechanical Drive, School of Mechanical and Architectural Engineering, Jianghan University, \\ 430050 Wuhan, P. O. B. 22\#, China \\ * Corresponding author, e-mail: 1253437676@qq.com
}

Received: 04 June 2018, Accepted: 16 July 2018, Published online: 14 June 2019

\begin{abstract}
Locomotive traction gear is the key component to power transmission and speed control in locomotive transmission system, which plays an important role in locomotive running speed and load-carrying torque. Considering that there is not universal rule for the method of modification of locomotive gear at present, in this paper, the tooth profile modification is considered with the combination of the increased contact ratio and the variation of the moment arm of action. Based on the principle of modification, according to the load direction after modification, the change rule of moment arm of action after modification is determined, and the interval range of tooth profile modification is also determined. Taking a certain locomotive traction gear as an example, the results obtained through the method of modification which based on combining moment arm of action variation with the increase of contact ratio and the method based on the traditional empirical formula are compared through finite element simulation respectively, on this account to verify the superiority of the theory of modification, which has important theoretical significance for profile modification of locomotive traction gear.
\end{abstract}

\section{Keywords}

locomotive traction gear, contact ratio, moment arm of action, modification coefficient, profile modification

\section{Introduction}

The traction gear which plays an important role in the locomotive running speed and load-carrying torque is also the core component of power transmission and speed control in locomotive transmission system. The meshing status of traction gear has a great influence on the locomotive vibration, noise and safety operation. Due to the influence of manufacturing, assembly and base pitch errors, unmodified gears may generate relatively large dynamic loads and impacts when engaged in and engaged out, the change in the logarithm of meshing teeth during the meshing process, the change of stiffness in a short period of time can also cause more serious incentives. In order to eliminate these negative effects and improve the meshing performance, many experts and scholars have conducted extensive research on tooth profile optimization techniques. Tavakoli and Houser (1986) used tooth profile modification as an effective tool to reduce gear meshing excitation, which turns out to be an effective way for eliminating the pound between meshing in and meshing out of gear teeth and minimizing fluctuations in gear transmission error. On the basis of the time-varying meshing stiffness, Beijing University of Science and Technology Li Wei systematically analyzes the effect of tooth profile modification parameters on the load distribution between teeth and determines the optimum modification parameters for tooth profile under single target conditions (Liu et al., 2018).

The methods of modification which used commonly are mainly based on a large number of experimental data, and most of them are based on empirical formulas. However, there is no unified basic rule being proposed and not possessed versatility. Therefore, this research aims at improving the bearing capacity by combining with manufacturing-factors. The tooth profile modification is mainly considered with the combination of the increase of contact ratio and moment arm of action variation, by means of finite element simulation, on this account to explore the technique of gear profile modification of high-speed and heavy-duty locomotives gear. 


\section{The Method of Modification Based on the Theory of Moment Arm of Action Variation}

\subsection{The Principle of Moment Arm of Action Variation in the Modification of Involute Cylindrical Gear}

According to the working environment of the traction gear and the characteristic requirements of high speed and heavy load, the ultimate goal of gear modification is to improve the carrying capacity of gears, which can be shown concretely to increase the length of the actual line of action as a result to increase the contact ratio. According to the principle of gear meshing, the driving gear must be rotated by a certain additional angle after modification to maintain contact with the driven gear, which makes the actual action position move over a segment of additional distance on the line of action, and biased toward the tangent point which is at line of action on the base circle. As shown in Fig. 1, taking the factors of manufacturing errors and assembly requirements into consideration, the driving gear needs to rotated by an angle $\delta$ to overcome the initial backlash on the tooth side to maintain contact with the driven gear, which makes the actual rotation angle of the gear different from the theoretical angle, the actual contact position changes, the length of the actual line of action $t$ increases to a certain extent. Due to the elastic deformation engendered by the load when in gear engagement, the rotation of the driving gear and the driven gear is relatively unevenly rotated, which is an important causation of the unsteadiness of the gear transmission. As shown in Fig. 2, after overcoming this deformation, the gear still needs to rotate a segment of additional angle $\theta$ to maintain contact with each other. At this time, the actual tooth profile moves along the line of action for a segment of additional distance to keep contact with the driven gear, and the contact position will move to a certain extent (Tang et al., 2016).

In order to meet the characteristics of constant transmission ratio when in working, the contact point of the tooth

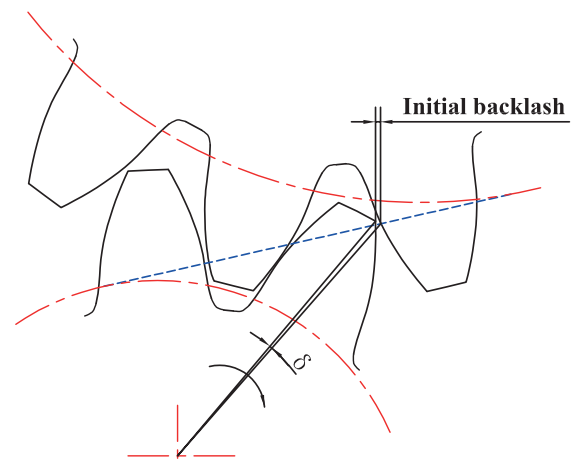

Fig. 1 Angle to rotate in order to overcome backlash

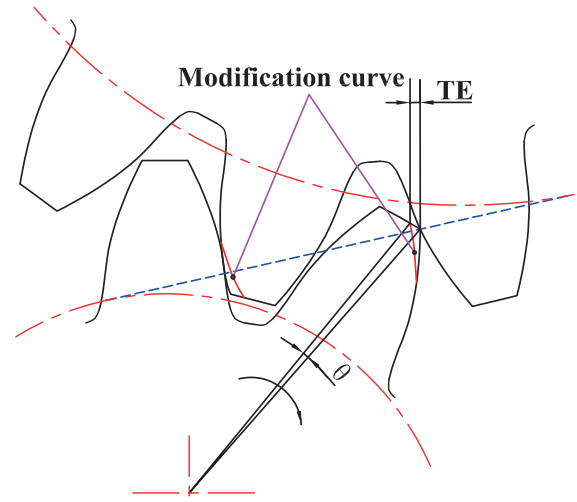

Fig. 2 Additional angle to rotate after modification

profile on the working side of the meshing gear is required to be on the line of action all the time. If more pairs of teeth are engaging simultaneously, the contact points must also be on the line of action, such as the contact points K and K', shown in Fig. 3. Since the modification does not change the transmission properties, the contact point after modification should also be on the line of action. It is considered that the part modified is the amount of deformation, and the amount of deformation in each section is relatively small, the angle $\theta$ for rotating of the gears to maintain contact

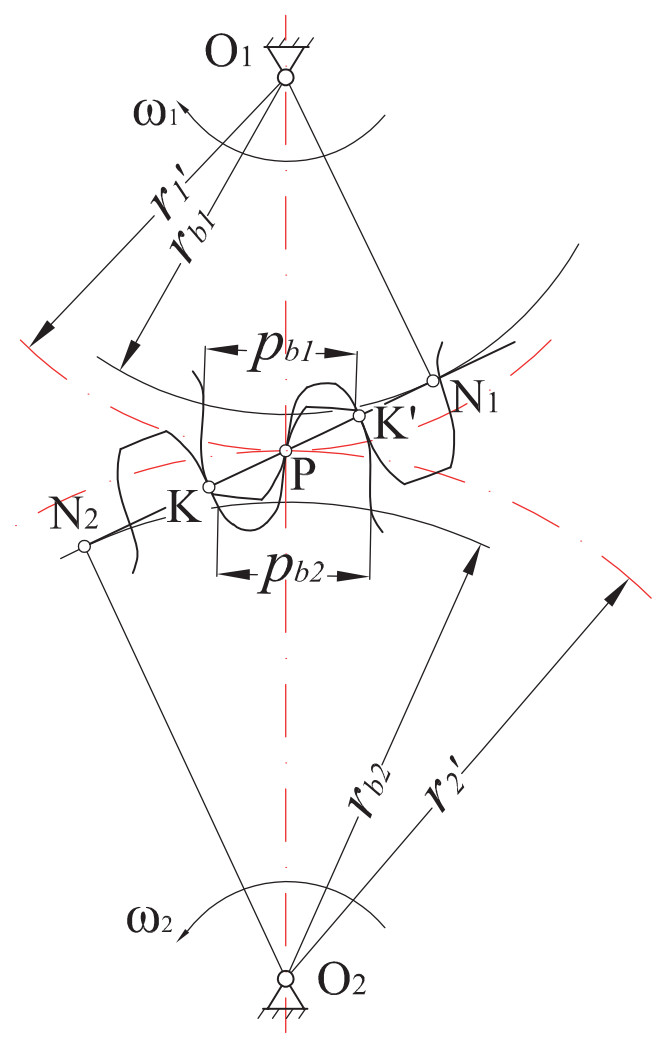

Fig. 3 Contact point on the line of action 
is relatively small accordingly, it can be approximately considered that the new contact point is still on the line of action. The length of line of action: $T E=r_{x} \sin (\theta) \approx r_{x} \theta$. Since the actual line of action has increased to a certain extent after modification, it can be known from the definition of contact ratio that the length of the actual line of action increases and the pitch $p_{b}$ of the basic pitch is constant, and the contact ratio is increased (Zhang, 2018).

\subsubsection{Curve Modification}

Since the tooth profile is no longer the standard involute after modification, instead, it is characterized by the profile of the modified curve, when the actual contact position is found, the direction of load is no longer along the direction of the line of action, and the moment arm of action also changes. The moment arm of action is directly related to the direction of load. Determining the direction of load is the necessary and sufficient condition to determine the size of moment arm of action. The common method for profile modification of gear is the modification of generatrix. There are many types of modification curves in the method of modification of generatrix. Owing to the orientations of force after modification determined by the radius of curvature and perpendicular to the tangent direction at the actual contact point, they are different for different types of modified curves. According to the force analysis of the gear structure, it can be seen that the quantity of gear moment arm of action and load is the torque $M$. As the starting point of modification is fixed and the amount of modification is 0 , when the modification curve is determined, the equation of gear modification curve can be determined simply by considering the value of the modification at the tip of the tooth.

As shown in Fig. 4, the layered modification based on elastic deformation can be achieved through the Do-Loop cycle statement, and each segment $\delta_{n}$ of the deformation is superimposed as the total amount of modification. Each section of the modification will result in the intersection into a deviation between the modification curve and the tip circle, at this time, a series of modification parameters including the orientation of force, the magnitude of the moment arm of action and load will change.

Generally speaking, the amount of modification in tip circle is proportionate to the contact ratio in theory. However when the maximum amount of modification in tip circle reaches a specific value or a specific range, the gear may no longer satisfy the contact fatigue strength or the bending fatigue strength in consideration

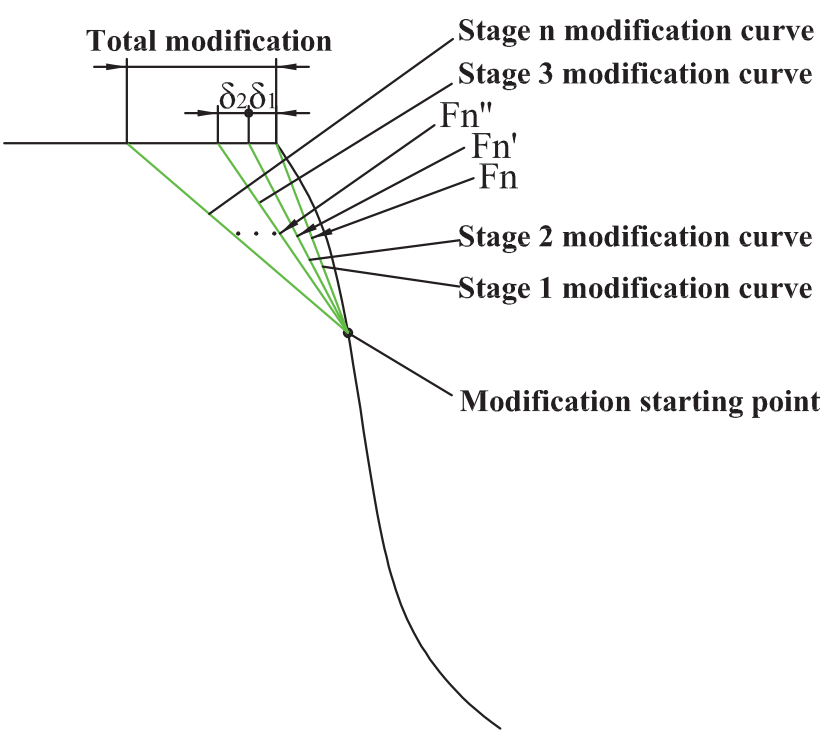

Fig. 4 Curve modification Diagram

of the safety factors under different working conditions. Therefore, the contact or bending fatigue strength is generally used as the design standard to calculate the amount of modification in tip circle, and the boundary condition for checking the modification curve is whether the static strength is satisfied. That is to say, the "degree" of the profile modification is generally achieved according to the reverse design, that is, considering the combination of the account of actual acting load and the boundary condition to solve the meshing deformation before modification, then tooth profile modification of gear is carried out by reverse eliminating material according to the amount of elastic deformation (Chen et al., 2018).

\subsubsection{The Determination of the Moment Arm of Action after Modification}

According to the principle of gear meshing, it is known that gears with addendum modification which are non-standard installed without gear profile modification is tangent to the pitch circle during the meshing process, the direction of generated normal force is always consistent with the direction of the line of action when the gear pair is in meshing, which is the same as the tangent direction of the base circle. When the method of modification of generatrix is used to modify the profile, the direction of normal force $F_{n}$ also changes due to the change in radius of curvature. Taking the straight-line modification as an example, as shown in Fig. 5, the first stage of the profile curve is to trim the involute tooth profile into a linear tooth profile mainly on the premise that the amount of modification in the tip circle is 0 . At this time, the direction of normal 


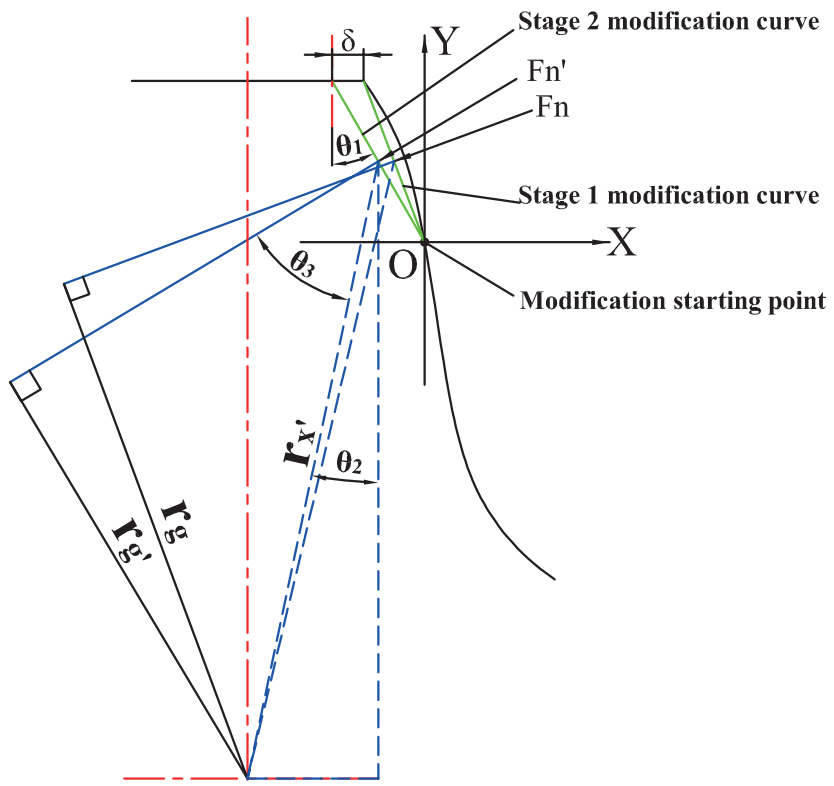

Fig. 5 The change regulation of moment arm of action after modified by straight line

force is no longer along the direction of the line of action and the moment arm of action $r_{g} \neq r_{b}$. Cartesian coordinate system XOY is established at the modification starting point. After the deformation $\delta$ which generated in gear meshing is modified, the modification curve evolves to the second stage. The value of $\theta_{1}$ can be obtained from the slope of the second stage modification curve. The value angle of $\theta_{2}$ can also be determined according to the height of contact point and the radius of contact point which is known based on the trigonometric function relationship, and the calculation formula is expressed as Eq. (1):

$\theta_{2}=\arccos \left(\frac{h_{x}^{\prime}}{r_{x}^{\prime}}\right)$

where $h_{x}^{\prime}$ is the actual height of contact point; $r_{x}^{\prime}$ is the actual radius of contact point.

When the load acts on the tip of the tooth, $\theta_{2}$ can be characterized as a function of the amount of modification in tip circle, expressed as Eq. (2):

$\theta_{2}=\arcsin \left(\frac{s_{a 1}-\delta}{r_{a 1}}\right)$

where $\delta$ is the amount of modification in tip circle, $s_{a 1}$ is half tooth thickness in tip circle, and $r_{a 1}$ is radius of tip.

Since the direction of normal force is perpendicular to the tangent direction of the modification curve, the equation of moment arm of action of actual contact position is expressed as Eq. (3):

$r_{g}^{\prime}=r_{x}^{\prime} \cdot \sin \left(90-\theta_{1}-\theta_{2}\right)$.

\subsubsection{Determination of Modification Section}

The distribution mode of gear modification can be roughly divided into three categories:

1. modifying at tip circle of the driving and driven gear;

2. only modifying at both tip and root circle of the driving gear without adjusting the driven gear;

3. modifying at both tip circle and root circle of driving gear and driven gear.

However, since root relief will increase the bending stress in teeth root and reduce bearing capacity of the gear, modifying at tip is mainly performed both on the driving and driven gear (tip relief), as shown in Fig. 6.

The purpose of tip relief is to reduce or even eliminate the impact which happens during engaging-in and engaging-out, so that the load can be smoothly transitioned, the stability of the meshing process can be improved, the vibration and noise can be reduced. The working conditions of the $\mathrm{CD}$ zone of action can be improved by tip relief on driving gear, the working condition of the $\mathrm{AB}$ zone of action can be improved by tip relief on driven gear.

Due to the abrupt change on load at the point of alternating transition between single and double tooth zone of action during gear meshing transmission, serious dynamic load and shocks will be generated. Therefore, the interval range of modification is from the tip to the point where the number of pairs of teeth is alternated.

\subsection{Time-Varying Meshing Stiffness}

\subsubsection{Analysis on Time-Varying Meshing Stiffness}

Since the technology of gear modification involves the amount of deformation, the influence of stiffness factors should be taken into account when calculating the amount of deformation, so in general, the time-varying meshing stiffness runs through entire calculation of modification. At present, numerical solutions for time-varying

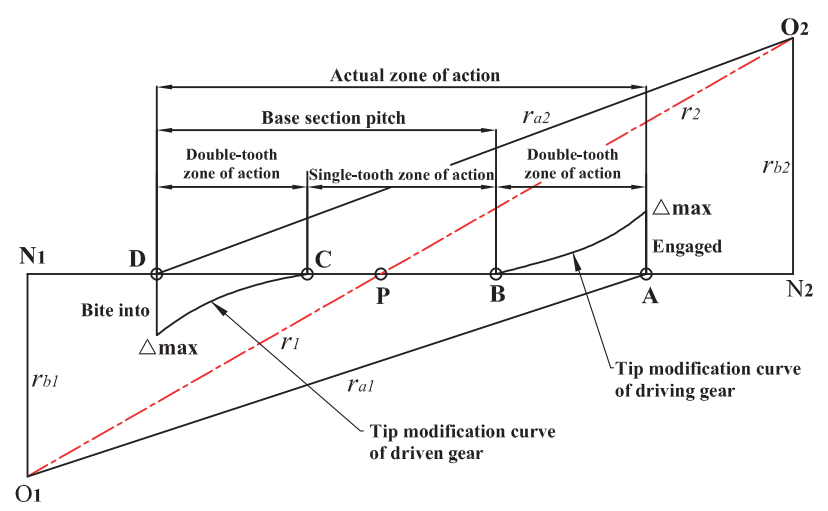

Fig. 6 Distribution of modification curves in line of action 
meshing stiffness include the Ishikawa formula and the Weber-Banaschck formula. Taking the Ishikawa formula as an example, taking into account the complex structure of the involute cylindrical gear, the Ishikawa formula converts the actual structure of gear into the structure of cantilever beam, which is finally simplified to a trapezoid plus a rectangle, as shown in Fig. 7, and then the stiffness of each part can be solved. However, the method above is mainly targeted for spur gear (Liu et al., 2018).

Due to the inclination of the contact line and the transmission characteristics of the non-uniform distribution of the load during the meshing transmission process, it is essentially determined that the helical gears cannot be referenced to the simplification of the spur gear into a $2 \mathrm{D}$ planar model. However, considering that the formation mechanism of the helical gear profile is similar to that of the spur gear, it is also an effective way to divide the helical gears along the width of the teeth into a number of spur gears with the same end face parameters, thin thickness and interlaced arrangement. The time-varying meshing stiffness of helical gears can be solved by combining the theory of variable cross-section cantilever with the concept of calculus summation in higher mathematics.

\subsubsection{The Influence of the Modification Coefficient on the Time-Varying Meshing Stiffness}

Considering the separability of the transmission in involute cylindrical gear, when the center distance for installation of the gear is not equal to the center distance of the standard gear, it is a case of installation which is non-standard. When the gear is mounted as shown in Fig. 8 (a), it is obvious that working pressure angle $\alpha^{\prime}$ is equal to the pressure angle $\alpha$, the center distance $\alpha$ is the standard center

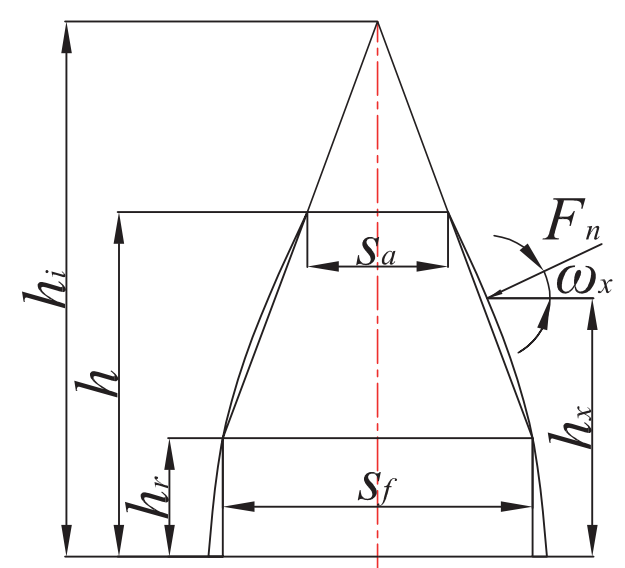

Fig. 7 Model of Ishikawa formula for solving meshing stiffness
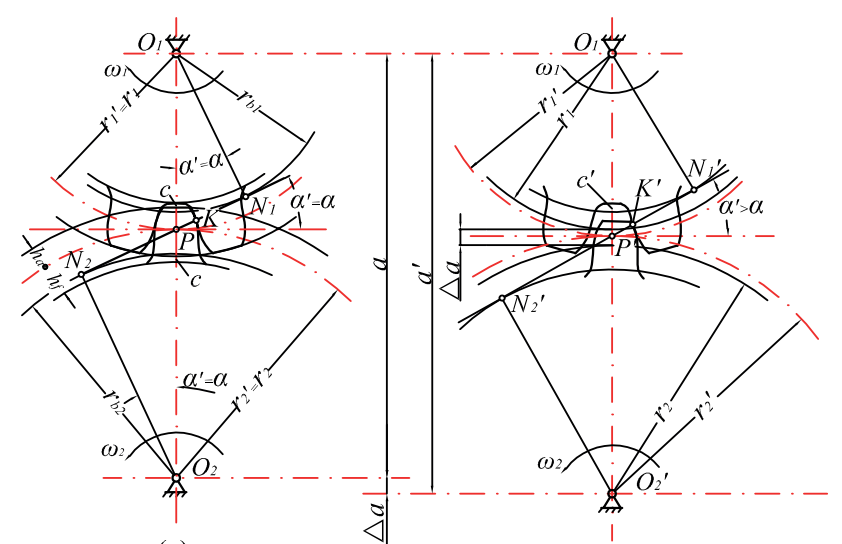

(b)

Fig. 8 Comparison and analysis of installation in gear with positive addendum modification and standard gears

(a) Standard gear mounting (b) Gear with positive addendum modification mounting

distance, when the gear adopts the non-standard installation as shown in Fig. 8 (b), the center distance $\alpha^{\prime}$ increases to a certain extent, at this time the working pressure angle $\alpha^{\prime}$ is larger than pressure angle $\alpha$. It is known from the separability of the gear that line of action of a pair of contact gear is the internal tangents of the base circles of two gears, and there is only a unique and fixed intersection point between the line of action and the gear center line, it also means the point which instantaneous center of speed is 0 . Since most locomotive traction gears are gear with positive addendum modification, the center distance for installation has increased to a certain extent relative to the standard gear. The length of actual line of action of the gear with positive addendum modification is shorter than that of the standard gear, but the actual working pressure angle increases to a certain extent, when the center distance of the gear with positive addendum modification is $\alpha^{\prime}$ the working pressure angle is expressed as Eq. (4):

$\alpha_{t}^{\prime}=\arccos \left(\frac{a}{a^{\prime}} \cos \left(\alpha_{n}\right)\right)$

where $\alpha$ is the center distance of reference circle, is also the center distance of the standard gear; $\alpha_{n}$ is the gear pressure angle.

The actual meshing interval is divided according to the boundary conditions of single and double tooth zone of action, and the change law of the working pressure angle of the gear with positive addendum modification must be taken into account when calculating the time-varying meshing stiffness (Chen et al., 2018). 


\subsection{Load Distribution between Teeth}

When the gear pair meshes in the single tooth zone of action during the process of meshing transmission, there is no load distribution, while in the double tooth zone of action, the problem of load distribution between the gear teeth can be converted from a dynamic point of view to the parallel spring which bearing load simultaneously. The load distribution factor can be determined by the ratio of elastic coefficient of parallel spring.

\subsection{Boundary Conditions for Designing and Checking of Modification}

\subsubsection{Boundary Conditions for Designing of Modification}

The boundary conditions of profile modification mainly include bending fatigue strength on root and contact fatigue strength on tip. When calculating the bending stress on root, it is usually assumed that the load exerts exactly on the tip and is only supported by a pair of teeth. The calculation of contact fatigue strength on tooth surface is usually represented by Hertz stress at node P. A large number of experimental data show that the result from this calculation method is partial to safety.

Formula for bending stress on root is expressed as Eq. (5):

$\sigma_{F 1,2}=\sigma_{F 0} K_{A} K_{V} K_{F \beta} K_{F \alpha}$

where $K_{A}$ is activity coefficient, $K_{V}$ is dynamic load coefficient, $K_{F \beta}$ is load distribution coefficient of tooth direction for bending calculation, $K_{F \alpha}$ is load distribution coefficient between teeth for bending calculation, $\sigma_{F 0}$ is the basic value of the root stress.

\subsubsection{Boundary Conditions for Checking of Modification}

Due to the need of large torque motor driving for locomotive operation, repeated load action or severe dynamic load and meshing impact will occur during operation, so that static strength verification is required.

Formula for maximum Static strength of bending stress on root is expressed as Eq. (6):

$\sigma_{F s t}=K_{V} K_{F \beta} K_{F \alpha} \frac{F_{c a 1}}{b m_{n}} Y_{F} Y_{S} Y_{\beta}$

where for gear with positive addendum modification, $d_{1}^{\prime}$ is the pitch diameter, $Y_{F}$ is the tooth profile coefficient at the dangerous section obtained by $30^{\circ}$ tangent method, $Y_{S}$ is the stress correction coefficient, and $Y_{\beta}$ is the helix angle coefficient calculated for bending strength, $F_{c a 1}$ is bearing capacity for calculation.
According to the actual working conditions of the gear, it is necessary to check the static strength with the 5 times torque under continuous working state. Defining the allowable static strength of contact and bending is $\sigma_{H G s t}$ and $\sigma_{F G s t}$. When the static strength of modified gear no longer satisfies the conditions of $\sigma_{H s t} \leq \sigma_{H G s t}$ or $\sigma_{F s t} \leq \sigma_{F G s t}$, it indicates that the equation of tooth modification curve is not satisfied with the working condition and should be recalculated.

\section{The Principle of Traditional Tooth Profile Modification}

The technology of traditional tooth profile modification is mainly based on three variables of maximum amount of modification, length of modification and modification curve, which are sequentially solved by empirical formula.

The maximum amount of modification is to counteract the interference of the tooth profile at the moment of meshing in and out of the gear. There are mainly two kinds of mainstream thinking in the calculation method. The first type is to determine the maximum amount of modification according to the deformation of the tooth when load acts on. The second type is to determine the maximum amount of shape modification according to the precision of gear manufacturing. Since the gear is an elastic body, its parameters such as contact, bending and shear deformation are difficult to determine, International Organization for Standardization formulas and elastic deformation formulas are commonly used (Yuan et al., 2017):

1. According to the ISO formula:

Such method mainly considers the working conditions of the gear box, the circumferential force, the tooth width, meshing stiffness and contact ratio. The maximum amount of modification of the tooth profile of driving and driven gears is defined as Eq. (7):

$$
z_{\max }=\frac{K_{A} F_{t} / b}{\varepsilon_{\alpha} C_{\gamma}}
$$

where: $F_{t}$ - circumferential force in reference circle; $b$ - face-width, unit is $\mathrm{mm} ; \varepsilon_{\alpha}$ - Transverse ratio; $C_{\gamma}$ - comprehensive stiffness of gear; meshing stiffness can be determined according to $C_{\gamma}=C_{1}\left(0.25+0.75 \varepsilon_{\alpha}\right)$.

2. Elastic deformation formula:

If some other error factors are neglected, the front pair of gear teeth reaches at the end of the actual line of action when it is at the starting point of the single-tooth zone of action, the elastic deformation of the gear pair at the terminal position is the maximum amount of modification on the top of the driving gear. 
Similarly, the terminal pair of gear teeth reaches at the starting point of the actual line of action when it is at the terminal point of the single-tooth zone of action, the elastic deformation of the gear pair at the starting position is the maximum amount of modification on the top of the driven gear. While in the actual process, the maximum modification is determined by combining the maximum amount of elastic deformation and thermal deformation which cause the interference and based on the manufacturing error of the gear and base pitch error. Calculation formula is expressed as Eq. (8):

$\left\{\begin{array}{l}\Delta_{\text {in }}=\delta_{B 1}=\delta_{D 1}+\delta_{D 2} \pm \Delta f_{b} \\ \Delta_{\text {out }}=\delta_{B 2}=\delta_{C 1}+\delta_{C 2} \pm \Delta f_{b}\end{array}\right.$

where: $\delta_{C 1}, \delta_{C 2}$ - The elastic deformation of the teeth of the driving and driven gear at the meshing alternating point $\mathrm{C} ; \delta_{D 1}, \delta_{D 2}$ - The elastic deformation of the teeth of the driving and driven gear at the meshing alternating point $\mathrm{D} ; \Delta f_{b}$ - base pitch error caused by gear manufacturing errors.

The length of modification is divided into long modification and short modification. Short modification should be able to ensure that the transverse ratio is more than 1 to effectively reduce the impact of intrusion. Long modification is generally used for helical gears with large spiral angles and large face-width because that if long modification has been taken, the load transition will be more stable when at the moment where single and double tooth meshing area is alternating. For traction gears, short modification is sufficient. The length of gear modification is generally determined based on empirical calculation formula which is determined by parameters such as center distance, working pressure angle, tip diameter and base diameter of the driving and driven gears, and length of transverse base pitch (Motahar et al., 2016).

There are many different types of modification curves. However, the vast majority are derived from basic form recommended by Walker: $\Delta=\Delta_{\max }(x / l)^{\beta}$. For the power exponent $\beta$, recommended values are generally between 1 and 2 to ensure that the load on the gear teeth changes slowly, so as to reduce sudden change of load and impact of meshing intrusion. Modification curve which commonly used is linear when $\beta=1 ; \beta$ of modification curve recommended by walker is $1.5 ; \beta$ of modification curve recommended by Hidaka, Terauchi and Fujii is 1.22 (Nie et al., 2016).

\section{Case Study and Experimental Reference}

In this paper, a certain type of locomotive traction gear is taken as an example to comparatively study the change of the stress in the condition of unmodified and modified through moment arm of action variation, so as to verify the rationality of the theory. Then the results obtained from the traditional method are compared to verify the superiority of this theory. The single-axis power of locomotive is $435 \mathrm{KW}$, and the rotation speed of high-speed motor is $602 \mathrm{r} / \mathrm{min}$. Table 1 shows the initial design parameters of the traction gear.

The processing technical specification of profile modification of involute gear determines that the range of modification is expressed in the form of tolerance zone. In general, after the maximum amount of modification on tip circle has been solved, the tolerance zone can be obtained when the tolerance of the tooth shape is taken into consideration. Through the analysis of the experimental data of this type of locomotive traction gear, it turns out that modification on tip circle of driving gear is between $40 \mu \mathrm{m} \sim 60 \mu \mathrm{m}$, and the modification on tip circle of driven gear is between $40 \mu \mathrm{m} \sim 70 \mu \mathrm{m}$, as shown in Fig. 9 is the K-shape diagram of profile modification. Profile modification is qualified when the maximum amount of modification on tip circle is located in this range.

Table 1 Partial initial design parameters

\begin{tabular}{lcc}
\hline Heading & Driving & Driven \\
\hline Module $m$ & \multicolumn{2}{c}{8.6} \\
Pressure angle $\alpha$ & \multicolumn{2}{c}{22.5} \\
Helix angle $\beta$ & \multicolumn{2}{c}{0} \\
Top gap coefficient $C^{*}$ & \multicolumn{2}{c}{0.35} \\
Face-width $b$ & 126 & \multicolumn{2}{c}{130} \\
Number of teeth $Z$ & 18 & \multicolumn{2}{c}{89} \\
Center distance $\alpha$ & \multicolumn{3}{c}{464} \\
Working pressure angle & \multicolumn{2}{c}{$23.64^{\circ}$} \\
Modification coefficient & 0.3914 & \multicolumn{2}{c}{0.07302} \\
\hline
\end{tabular}

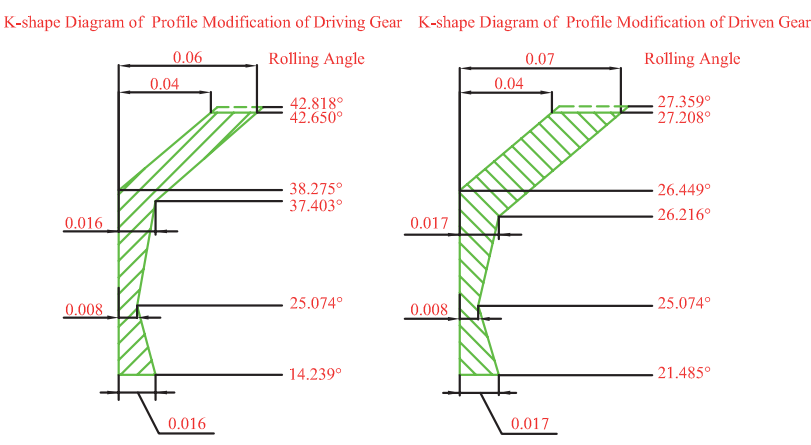

Fig. 9 K-shape diagram of profile modification 


\section{Demonstration of Result Data}

Through the above method which is according to the theory of moment arm of action variation to conduct profile modification. When the method of modification based on straight line is adopted, with the modification starting point as the origin of the local coordinate, the equation of modification curve obtained is as follows:

- Tip relief curve of driving gear is expressed as Eq. (9):

$$
y_{1}=49.097\left(\frac{x}{7.61}\right) \text {. }
$$

- Tip relief curve of driven gear is expressed as Eq. (10):

$$
y_{2}=54.656\left(\frac{x}{5.049}\right) \text {. }
$$

At this time, the maximum amount of modification in tip circle of driving and driven gear is within the range of the experimental tolerance zone. It is found that the profile error of driving gear is $0.016 \mathrm{~mm}$. When this factor is taken into consideration, the maximum value of tolerance belt of driving gear is $0.065 \mathrm{~mm}$, which is $8.1 \%$ of the difference between the existing data of $0.06 \mathrm{~mm}$, which belongs to the desirable range. The contact ratio has increased from 1.4544 to 1.4817 .

When calculating with the theory of traditional modification (ISO formula is taken as an example), the maximum amount of modification in the tip is $32.218 \mu \mathrm{m}$. The length of modification is $5.655 \mathrm{~mm}$ when short modification is adopted.

The results obtained through the two modification theories are compared with the unmodified gear model through the finite element simulation. The Mises stress contour plot obtained through the theory of moment arm of action variation is shown as Fig. 10. The result obtained from traditional formula is shown as Fig. 11. The Mises stress contour plot of the unmodified gear is shown as Fig. 12. When the load exerts exactly on a pair of gear and the contact position is as shown, the maximum equivalent stress obtained through the theory of modification based on moment arm of action variation is $448.4 \mathrm{Mpa}$ and the maximum equivalent stress obtained through the theory of modification based on traditional experience formula is $465.17 \mathrm{Mpa}$, while the maximum equivalent stress of unmodified gears is $489.15 \mathrm{Mpa}$. Through comparative analysis, it can be seen that the stress after modification with the theory of moment arm of action variation is reduced by $8.33 \%$ compared with that before modification, and reduced by $3.6 \%$ compared with that after

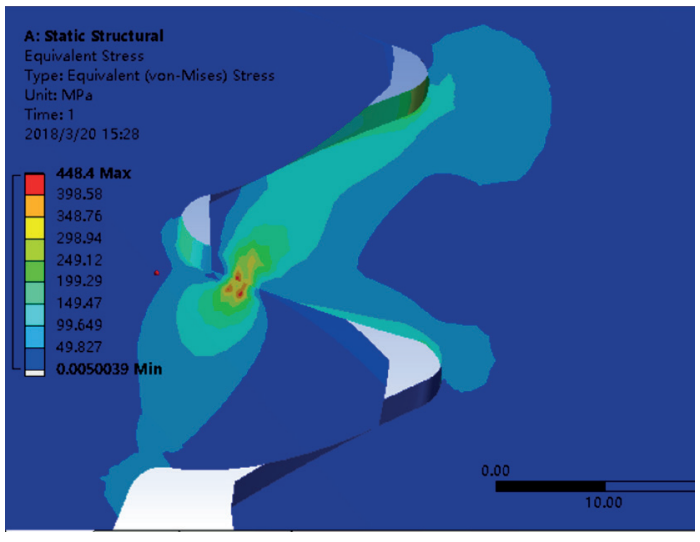

Fig. 10 The Mises stress contour plot obtained through the theory of moment arm of action variation

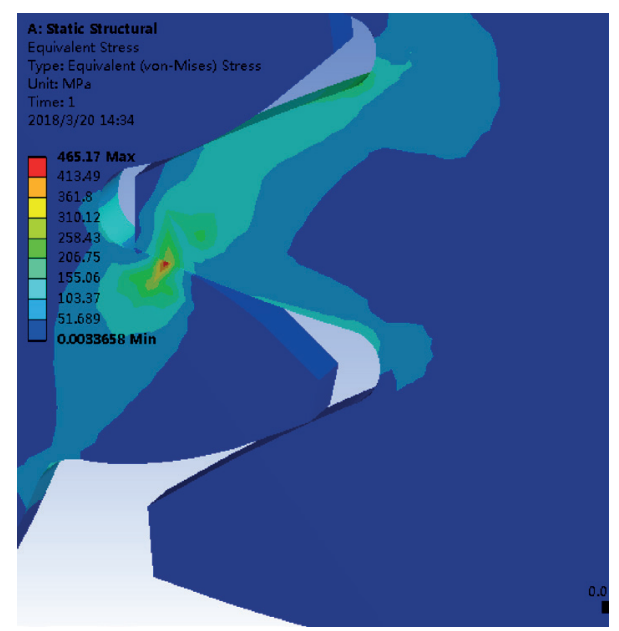

Fig. 11 The Mises stress contour plot obtained through the theory of traditional formula

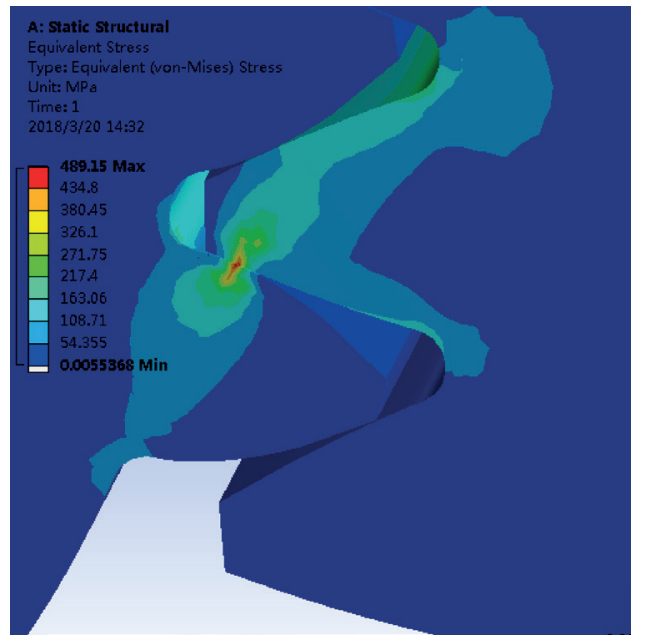

Fig. 12 The Mises stress contour plot before modification

modification with the theory of traditional experience formula. The research shows that the modification theory of moment arm of action variation has very good effect on modification on locomotive traction gear. 


\section{Conclusion}

1. The tooth profile modification is considered with the combination of the increased contact ratio and moment arm of action variation. Based on principle of modification, according to the load direction after profile modification, the change rule of moment arm of action after modification is determined. Taking a certain traction gear as an example, it is shown that the interval range of tooth profile modification belongs to the desirable range.

2. Through finite element simulation calculation, the results show that the method of tooth profile

\section{References}

Chen, H., Zhu, Y., Mao, J., Wang, X. (2018) "Influence of Tooth Profile Modification on Design Transmission Error of Involute Spur Gear", Journal of Huazhong University of Science and Technology (Natural Science Edition), 46(3), pp. 91-97. (in Chinese) https://doi.org/10.13245/j.hust.180317

Liu, W., Li, R., Zhang, J., Lin, T., Yang, Y. (2018) "Study on Correction Algorithm of Time-varying Mesh Stiffness of Helical Gears and its Influencing Factors", Journal of Hunan University (Natural Sciences), 45(2), pp. 1-10. (in Chinese) https://doi.org/10.16339/j.cnki.hdxbzkb.2018.02.01

Motahar, H., Samani, F. S., Molaie, M. (2016) "Nonlinear vibration of the bevel gear with teeth profile modification", Nonlinear Dynamics, 83(4), pp. 1875-1884.

https://doi.org/10.1007/s11071-015-2452-z

Nie, S., Hu, J., Li, B., Chen, D., Mei, J., Wu, J. (2016) "The Modification Optimization Analysis of Cycloid Pin Gear Tooth Profile of RV Reducer", Machine Design \& Research, 32(6), pp. 49-52. (in Chinese)

https://doi.org/10.13952/j.cnki.jofmdr.2016.0229 modification based on moment arm of action variation can greatly reduce the bending stress of the gear and compared with the traditional method of modification to highlight its superiority. It verifies that this theory of modification has a good effect on the traction gear profile modification of high-speed and heavy-duty locomotives.

\section{Acknowledgement}

The project presented in this article is supported by Science and Technology Research and Development Project of China Railway (No. 2017J009-H).

Tang, Z., Tang, S., Sun, J., Yan, L. (2016) "Traction Gear Modification and Noise Reduction Design on SS8-II Locomotive", Science Technology and Engineering, 16(8), pp. 201-205. (in Chinese) https://doi.org/10.3969/j.issn.1671-1815.2016.08.033

Tavakoli, M. S., Houser, D. R. (1986) "Optimum Profile Modifications for the Minimization of Static Transmission Errors of Spur Gears", Journal of Mechanisms, Transmissions and Automation in Design, 108(1), pp. 86-90. https://doi.org/10.1115/1.3260791

Yuan, Y., Shao, N., Lu, B., Zhang, L. (2017) "Research of Involute Gear Modification Curve Based on Finite Element Contact Analysis", Journal of Mechanical Transmission, 41(4), pp. 34-37. (in Chinese) https://doi.org/10.16578/j.issn.1004.2539.2017.04.008

Zhang, J. (2018) "Transverse Contact Ratio Calculation of Profile Modification Gear", Machine Design \& Research, 34(1), pp. 91-93. (in Chinese) https://doi.org/10.13952/j.cnki.jofmdr.2018.0021 